\title{
Existence of global attractors and gradient property for a class of non local evolution equations
}

\author{
Antônio Luiz Pereira ${ }^{1}$ \\ Instituto de Matemática e Estatística-USP, \\ Rua do Matão, 1010, Cidade Universitária, São Paulo-SP, Brasil. \\ E-mail address: alpereir@ime.usp.br
}

\section{Severino Horácio da Silva ${ }^{2}$}

Unidade Acadêmica de Ciências Exatas e da Natureza/CFP/UFCG,

Rua Sérgio Murilo de Figueredo, s/n, CEP 58900-000, Casas Populares, Cajazeiras-PB, Brasil.

E-mail address: horacio@cfp.ufcg.edu.br and horaciousp@gmail.com

\begin{abstract}
In this work we prove the existence of a compact global attractor for the flow of the equation

$$
\frac{\partial m(r, t)}{\partial t}=-m(r, t)+g(\beta J * m(r, t)+\beta h), \quad h, \beta \geq 0,
$$

in $L^{2}\left(S^{1}\right)$. We also give uniform estimates on the size of the attractor and show that the flow is gradient.

AMS subject classification: $34 \mathrm{G} 20,47 \mathrm{H} 15$.

Keywords:Global Attractor; Lyapunov Functional; Gradient Flow.
\end{abstract}

\section{Introduction}

We consider here the non local evolution equation

$$
\frac{\partial m(r, t)}{\partial t}=-m(r, t)+g(\beta J * m(r, t)+\beta h),
$$

where $m(r, t)$ is a real function on $\mathbb{R} \times \mathbb{R}_{+}, h$ and $\beta$ are non negative constants and $J \in C^{1}(\mathbb{R})$ is a non negative even function supported in the

\footnotetext{
${ }^{1}$ Partially supported by CNPq-Brazil grants 2003/11021-7, 03/10042-0.

${ }^{2}$ Partially supported by CNPq-Brazil grant 141882/2003-4.
} 
interval $[-1,1]$ and integral equal to 1 . The $*$ above denotes convolution product, namely:

$$
(J * m)(x)=\int_{\mathbb{R}} J(x-y) m(y) d y .
$$

An equilibrium of (1.1) is a solution for (1.1) that is constant with respect to $t$, that is, $m$ satisfies

$$
m(r)=g(\beta J * m(r)+\beta h) .
$$

There are several works in the literature dedicated to the analysis of the particular case of (1.1) where $g \equiv \tanh$, that is

$$
\frac{\partial m(r, t)}{\partial t}=-m(r, t)+\tanh (\beta J * m(r, t)+\beta h) .
$$

In this case, if $\beta \leq 1$, equation (1.2) has only one (stable) equilibrium, (see [9] and [11]). If $\beta>1$ there is $h^{*}$, implicitly defined by equation (1.3) below, such that, for $0 \leq h<h^{*}$, equation (1.2) has three spatially homogeneous equilibria, $m_{\beta}^{-}, m_{\beta}^{0}, m_{\beta}^{+}$, each of which is identically equal to one of the roots of the equation

$$
m_{\beta}=\tanh \left(\beta m_{\beta}+\beta h\right) .
$$

In [7], the existence and uniqueness (modulo translation) of a travelling front connecting the equilibria $m_{\beta}^{-}$and $m_{\beta}^{+}$is proved. In [9], the existence of a non-homogeneous stationary solution referred to as the "bump" is proved for $h$ "sufficiently close" to 1 . In [11], the existence of a such solution is established for $0<h<h^{*}$.

The existence and uniqueness (modulo translation) of an equilibrium for (1.2) which tends asymptotically to $\pm m_{\beta}^{+}$, referred to as the "instanton" is proved in [8] and [10] for the case $h=0$.

In [1], the existence of a global attractor for (1.2) is proved for the case of bounded domain and $h=0$.

We now collect the conditions on $g$ which will be used used as hypotheses along the paper and indicate the points where each one is needed.

(H1) The function $g: \mathbb{R} \rightarrow \mathbb{R}$, is globally Lipschitz, that is, there exists a positive constant $k_{1}$ such that

$$
|g(x)-g(y)| \leq k_{1}|x-y|, \quad \forall x, y \in \mathbb{R} .
$$

In particular, there exist non negative constants $k_{2}$ and $k_{3}$ such that

$$
|g(x)| \leq k_{2}|x|+k_{3}, \quad \forall x \in \mathbb{R} .
$$

(H2) The function $g \in C^{1}(\mathbb{R})$ and $g^{\prime}$ is locally Lipschitz. 
(H3) There exist non negative constants $k_{4}$ and $k_{5}$, such that

$$
\left|g^{\prime}(x)\right| \leq k_{4}|x|+k_{5}, \quad \forall x \in \mathbb{R} .
$$

(Observe that if (H1) and (H2) hold then (H3) also holds with $k_{4}=0$ and $k_{5}=k_{1}$.

(H4) The function $g$ has positive derivative. In particular it is increasing.

(H5) There exists $a>0$ such that, for all $x \in \mathbb{R},|g(x)|<a$. In particular, when $a<\infty$ (1.4) holds with $k_{2}=0$ and $k_{3}=a$.

(H6) The function $g^{-1}$ is continuous in $(-a, a)$ and the function

$$
f(m)=-\frac{1}{2} m^{2}-h m-\beta^{-1} i(m), m \in[-a, a],
$$

where $i$ is defined by

$$
i(m)=-\int_{0}^{m} g^{-1}(s) d s, m \in[-a, a],
$$

has a global minimum $\bar{m}$ in $(-a, a)$.

This paper is organized as follows. In Section 2 we prove that, under hypothesis (H1), (1.1) (restricted to $\left.P_{2 \tau}\right)$ ) generates a flow in $L^{2}\left(S^{1}\right)$, which is of class $C^{1}$ if one also assumes (H2). Section 3 is dedicated to the proof of existence of the global attractor, generalizing some results of [1], where the case $h=0$ was considered. This is done using hypotheses (H1) and (H3) (we don't need to assume (H2) at this point). In Section 4, we prove a comparison result under the hypotheses $(\mathbf{H 1})$ and $(\mathbf{H} 4)$, generalizing Theorem 2.7 of [8]. Assuming also (H3) and (H5) (with $a<0$ ) we prove an uniform estimate for the attractor. Finally, in Section 5 assuming (H6), we exhibit a continuous Lyapunov functional for the flow of (1.1), and as used it to prove that, under hypotheses (H1), (H3), (H4), (H5) and (H6), the flow is gradient in the sense of [5]. As consequence, the global attractor coincides with the unstable manifolds of the equilibria.

\section{Well posedness in $L^{2}\left(S^{1}\right)$}

The Cauchy problem for equation (1.1) in the space of continuous bounded functions, $C_{b}(\mathbb{R})$, with the sup norm is well posed, since the function given by the right hand side of (1.1) is uniformly Lipschitz in this space, (see [2] and [3]).

It is an easy consequence of the uniquennes theorem that the subspace $\mathbb{P}_{2 \tau}$ of $2 \tau$ periodic functions is invariant, 
We considerer here the equation (1.1) restricted to the $\mathbb{P}_{2 \tau}, \tau>1$. As we will see below, this leads naturally to the consideration of a flow in $L^{2}\left(S^{1}\right)$, where $S^{1}$ denotes the unit sphere.

Now, if $\tau>1$ is a given positive number, we define $J^{\tau}$ as the $2 \tau$ periodic extension of the restriction of $J$ to interval $[-\tau, \tau]$. It is then easy to show that, if $u \in \mathbb{P}_{2 \tau}$, then

$$
(J * u)(x)=\int_{-\tau}^{\tau} J^{\tau}(x-y) u(y) d y
$$

In view of the (2.5), the equation (1.1), restricted to $\mathbb{P}_{2 \tau}$, with $\tau>1$, can be written as

$$
\frac{\partial m(x, t)}{\partial t}=-m(x, t)+g\left(\beta \int_{-\tau}^{\tau} J^{\tau}(x-y) m(y, t) d y+\beta h\right) .
$$

Define $\varphi: \mathbb{R} \rightarrow S^{1}$ by

$$
\varphi(x)=e^{i \frac{\pi}{\tau} x}
$$

and, for $u \in \mathbb{P}_{2 \tau}, v: S^{1} \rightarrow \mathbb{R}$ by

$$
v(\varphi(x))=u(x)
$$

In particular, we write $\widetilde{J}(\varphi(x))=J^{\tau}(x)$. Then we have the following result, whose simple proof is omitted.

Proposition 2.1. The function $u(x, t)$ is a $2 \tau$ periodic solution of (1.1) if and only if $v(w, t)=u\left(\varphi^{-1}(w), t\right)$ is a solution of

$$
\frac{\partial m(w, t)}{\partial t}=-m(w, t)+g(\beta \widetilde{J} * m(w, t)+\beta h),
$$

where, now, (*) denote convolution in $S^{1}$, that is

$$
(\widetilde{J} * m)(w)=\int_{S^{1}} \widetilde{J}\left(w \cdot z^{-1}\right) m(z) d z
$$

and $d z=\frac{\tau}{\pi} d \theta$, where $d \theta$ denote integration with respect to arc length.

From now on we will write $J$ instead of $\widetilde{J}$ for simplicity.

Proposition 2.2. Suppose that the hypothesis (H1) holds. Then the function

$$
F(u)=-u+g(\beta J * u+\beta h)
$$

is uniformly Lipschitz in $L^{2}\left(S^{1}\right)$.

São Paulo J.Math.Sci. 2, 1 (2008), 1-20 
Proof From (H1) and the triangle inequality, we obtain

$$
\begin{aligned}
\|F(m)-F(u)\|_{L^{2}} & = \\
& =\|-(m-u)+g(\beta J * m+\beta h)-g(\beta J * u+\beta h)\|_{L^{2}} \\
& \leq\|m-u\|_{L^{2}}+k_{1}\|\beta(J * m)-\beta(J * u)\|_{L^{2}} \\
& =\|m-u\|_{L^{2}}+k_{1} \beta\|J *(m-u)\|_{L^{2}} .
\end{aligned}
$$

But, from Young's inequality, (see [4]),

$$
\begin{aligned}
\|J *(m-u)\|_{L^{2}} & \leq\|J\|_{L^{1}}\|m-u\|_{L^{2}} \\
& =\|m-u\|_{L^{2}} .
\end{aligned}
$$

Thus

$$
\|F(m)-F(u)\|_{L^{2}} \leq\left(1+k_{1} \beta\right)\|m-u\|_{L^{2}},
$$

which concludes the proof.

From Proposition 2.2, it follows that the Cauchy problem for (2.6) is well posed in $L^{2}\left(S^{1}\right)$ with a unique global solution, (see [2] and [3]). More precisely, we have

Corollary 2.3. Equation (2.6) has a unique solution for any initial condition in $L^{2}\left(S^{1}\right)$, which is globally defined.

The following result has been proven in [12].

Proposition 2.4. Let $X$ and $Y$ be normed linear spaces, $F: X \rightarrow Y$ a map and suppose that the Gateaux derivative of $F, D F: X \rightarrow \mathcal{L}(X, Y)$ exists and is continuous at $x \in X$. Then the Frechet derivative $F^{\prime}$ of $F$ exists and is continuous at $x$.

Remark 2.5. If $u \in L^{2}\left(S^{1}\right)$, then

$$
|(J * u)(w)| \leq \sqrt{2 \tau}\|J\|_{\infty}\|u\|_{L^{2}}, \forall w \in S^{1} .
$$

In fact,

$$
\begin{aligned}
|(J * u)(w)| & \leq \int_{S^{1}}\left|J\left(w z^{-1}\right)\right||u(z)| d z \\
& \leq \int_{S^{1}}\|J\|_{\infty}|u(z)| d z
\end{aligned}
$$

and the estimate follows from Hölder's inequality, (see [2]).

Proposition 2.6. Assume that the hypotheses (H1) and (H2) hold. Then the function

$$
F(u)=-u+g(\beta J * u+\beta h)
$$


is continuously Frechet differentiable in $L^{2}\left(S^{1}\right)$ with derivative given by

$$
F^{\prime}(u) v=-v+g^{\prime}(\beta J * u+\beta h) \beta(J * v) .
$$

Proof By a simple computation, using the hypothesis (H1), it follows that the Gateaux's derivative of $F$ is given by

$$
D F(u) v=-v+g^{\prime}(\beta J * u+\beta h) \beta(J * v) .
$$

Now, note that for each $u \in L^{2}\left(S^{1}\right)$, due to linearity of the convolution, $D F(u)$ is a linear operator. Furthermore

$$
\|D F(u) v\|_{L^{2}} \leq\|v\|_{L^{2}}+\left\|g^{\prime}(\beta J * u+\beta h) \beta(J * v)\right\|_{L^{2}} .
$$

But, from (2.7), we have

$$
|\beta(J * v)(w)| \leq \sqrt{2 \tau} \beta\|J\|_{\infty}\|v\|_{L^{2}}, \forall w \in S^{1}
$$

and, from $(\mathrm{H} 2)$

$$
\int_{S^{1}}\left|g^{\prime}(\beta(J * u)(w)+\beta h)\right|^{2} d w=L<\infty .
$$

Hence

$$
\begin{aligned}
\| g^{\prime}(\beta J * u+\beta h) & \beta(J * v) \|_{L^{2}}^{2}= \\
& =\int_{S^{1}}\left|g^{\prime}(\beta(J * u)(w)+\beta h)\right|^{2} \beta^{2}|(J * v)(w)|^{2} d w \\
& \leq \int_{S^{1}}\left|g^{\prime}(\beta(J * u)(w)+\beta h)\right|^{2} \beta^{2} 2 \tau\|J\|_{\infty}^{2}\|v\|_{L^{2}}^{2} d w \\
& =\beta^{2} 2 \tau\|J\|_{\infty}^{2}\|v\|_{L^{2}}^{2} \int_{S^{1}}\left|g^{\prime}(\beta(J * u)(w)+\beta h)\right|^{2} d w \\
& =L \beta^{2} 2 \tau\|J\|_{\infty}^{2}\|v\|_{L^{2}}^{2} .
\end{aligned}
$$

Thus

$$
\left\|g^{\prime}(\beta J * u+\beta h) \beta(J * v)\right\|_{L^{2}} \leq \sqrt{L} \beta \sqrt{2 \tau}\|J\|_{\infty}\|v\|_{L^{2}} .
$$

Therefore

$$
\|D F(u) v\|_{L^{2}} \leq\left(1+\sqrt{L 2 \tau} \beta\|J\|_{\infty}\right)\|v\|_{L^{2}} .
$$

Furthermore, $D F$ is a continuous operator. In fact

$$
\begin{aligned}
& \left\|D F\left(u_{1}\right) v-D F\left(u_{2}\right) v\right\|_{L^{2}}= \\
& \quad=\left\|\left[g^{\prime}\left(\beta J * u_{1}+\beta h\right)-g^{\prime}\left(\beta J * u_{2}+\beta h\right)\right] \beta(J * v)\right\|_{L^{2}} .
\end{aligned}
$$

São Paulo J.Math.Sci. 2, 1 (2008), 1-20 
Keeping $u_{1} \in L^{2}\left(S^{1}\right)$ fixed and letting $u_{2} \rightarrow u_{1}$ in $L^{2}\left(S^{1}\right)$ it follows, from (2.7), that $\left(\beta J * u_{2}+\beta h\right)$ is in a ball of $L^{\infty}\left(S^{1}\right)$ centered in $\left(\beta J * u_{1}+\beta h\right)$. Thus, using hypothesis (H2), there exists a constant $M>0$ such that

$$
\left|g^{\prime}\left(\beta J * u_{1}+\beta h\right)(w)-g^{\prime}\left(\beta J * u_{2}+\beta h\right)(w)\right| \leq M \beta\left|J *\left(u_{1}-u_{2}\right)(w)\right| .
$$

Using this last estimative and (2.7), we obtain

$$
\begin{aligned}
\| D F\left(u_{1}\right) v & -D F\left(u_{2}\right) v \|_{L^{2}}=\left(\int_{S^{1}} \mid g^{\prime}\left(\beta J * u_{1}+\beta h\right)(w)-\right. \\
& \left.-\left.g^{\prime}\left(\beta J * u_{2}+\beta h\right)(w)\right|^{2} \beta^{2}|(J * v)(w)| d w\right)^{\frac{1}{2}} \\
& \leq\left(\int_{S^{1}} M^{2} \beta^{2}\left|J *\left(u_{1}-u_{2}\right)(w)\right|^{2} \beta^{2}|(J * v)(w)|^{2} d w\right)^{\frac{1}{2}} \\
& \leq M \beta^{2} 2 \tau \sqrt{2 \tau}\|J\|_{\infty}^{2}\left\|u_{1}-u_{2}\right\|\|v\|_{L^{2}} .
\end{aligned}
$$

It follows from Proposition 2.4 that $F$ is Frechet differentiable with continuous derivative in $L^{2}\left(S^{1}\right)$.

Remark 2.7. Since the right-hand side of (2.6) is a $C^{1}$ function, the flow generated by (2.6) is $C^{1}$ with respect to initial conditions, (see [6]).

\section{Existence of a global attractor}

We prove, in this section, the existence of a global maximal invariant compact set $\mathcal{A} \subset L^{2}\left(S^{1}\right)$ for the flow of (2.6), which attracts each bounded set of $L^{2}\left(S^{1}\right)$ (the global attractor, see [5] and [13]).

We recall that a set $\mathcal{B} \subset L^{2}\left(S^{1}\right)$ is an absorbing set for the flow $T(t)$ if, for any bounded set $C \subset L^{2}\left(S^{1}\right)$, there is a $t_{1}>0$ such that $T(t) C \subset \mathcal{B}$ for any $t \geq t_{1}$.

The following result was proven in [13]

Theorem 3.1. Let $X$ be a Banach space and $T(t)$ a semigroup on $X$. Assume that, for every $t, T(t)=T_{1}(t)+T_{2}(t)$ where the operators $T_{1}(\cdot)$ are uniformly compact for $t$ large, that is, for every bounded set $B$ there exists $t_{0}$, which may depend on $B$, such that

$$
\bigcup_{t \geq t_{0}} T_{1}(t) B
$$

is relatively compact in $X$ and $T_{2}(t)$ is a continuous mapping from $X$ into itself such that the following holds: For every bounded set $C \subset X$,

$$
r_{c}(t)=\sup _{\varphi \in C}\left\|T_{2}(t) \varphi\right\|_{X} \rightarrow 0 \quad \text { as } \quad t \rightarrow \infty .
$$


Assume also that there exists an open set $\mathcal{U}$ and bounded subset $\mathcal{B}$ of $\mathcal{U}$ such that $\mathcal{B}$ is absorbing in $\mathcal{U}$. Then the $\omega$-limit set of $\mathcal{B}, \mathcal{A}=\omega(\mathcal{B})$, is a compact attractor which attracts the bounded sets of $\mathcal{U}$. It is the maximal bounded attractor in $\mathcal{U}$ (for the inclusion relation). Furthermore, if $\mathcal{U}$ is convex and connected, then $\mathcal{A}$ is connected.

Lemma 3.2. Assume that the hypothesis (H1) holds and $k_{2} \beta<1$. Then the ball of radius $\frac{2 \sqrt{2 \tau}\left(k_{2} \beta h+k_{3}\right)}{1-k_{2} \beta}$ is an absorbing set for the flow $T(t)$ generated by (2.6).

Proof If $u(w, t)$ is a solution of (2.6) with initial condition $u(w, 0)$ then, by the variation of constants formula

$$
u(w, t)=e^{-t} u(w, 0)+\int_{0}^{t} e^{-(t-s)} g(\beta(J * u(w, s)+h)) d s .
$$

Thus

$$
\begin{aligned}
& \frac{d}{d t} \int_{S^{1}}|u(w, t)|^{2} d w= \\
& \quad=-2 \int_{S^{1}} u^{2}(w, t) d w+2 \int_{S^{1}} u(w, t) g(\beta J * u(w, t)+\beta h) d w .
\end{aligned}
$$

But, by Hölder inequality

$$
\begin{aligned}
& \int_{S^{1}} u(w, t) g(\beta J* u(w, t)+\beta h) d w \leq \\
& \leq\|u(\cdot, t)\|_{L^{2}}\left(\int_{S^{1}}(g(\beta J * u(w, t)+\beta h))^{2} d w\right)^{\frac{1}{2}} .
\end{aligned}
$$

Using (1.4) and Young's inequality in the right-hand side of the inequality above, we obtain

$$
\begin{array}{rl}
\int_{S^{1}} & u(w, t) g(\beta J * u(w, t)+\beta h) d w \leq\|u(\cdot, t)\|_{L^{2}} * \\
& *\left[k_{2} \beta\left(\int_{S^{1}}(J * u(w, t))^{2} d w\right)^{\frac{1}{2}}+\left(\int_{S^{1}}\left(k_{2} \beta h+k_{3}\right)^{2} d w\right)^{\frac{1}{2}}\right] \\
& \leq\|u(\cdot, t)\|_{L^{2}}\left[k_{2} \beta\|J\|_{L^{1}}\|u(\cdot, t)\|_{L^{2}}+\sqrt{2 \tau}\left(k_{2} \beta h+k_{3}\right)\right] \\
& =\|u(\cdot, t)\|_{L^{2}}\left[k_{2} \beta\|u(\cdot, t)\|_{L^{2}}+\sqrt{2 \tau}\left(k_{2} \beta h+k_{3}\right)\right] .
\end{array}
$$

São Paulo J.Math.Sci. 2, 1 (2008), 1-20 
Thus

$$
\begin{aligned}
& \frac{d}{d t}\|u(\cdot, t)\|^{2} \leq \\
& \quad \leq-2\|u(\cdot, t)\|_{L^{2}}^{2}+2 k_{2} \beta\|u(\cdot, t)\|_{L^{2}}^{2}+2 \sqrt{2 \tau}\left(k_{2} \beta h+k_{3}\right)\|u(\cdot, t)\|_{L^{2}} \\
& \quad=2\|u(\cdot, t)\|_{L^{2}}^{2}\left[-1+k_{2} \beta+\frac{\sqrt{2 \tau}\left(k_{2} \beta h+k_{3}\right)}{\|u(\cdot, t)\|_{L^{2}}}\right] .
\end{aligned}
$$

Since $k_{2} \beta<1$, let $\varepsilon=1-k_{2} \beta>0$. Then, while $\|u(\cdot, t)\|_{L^{2}} \geq \frac{2 \sqrt{2 \tau}\left(k_{2} \beta h+k_{3}\right)}{\varepsilon}$, we have

$$
\begin{aligned}
\frac{d}{d t}\|u(\cdot, t)\|_{L^{2}}^{2} & \leq 2\|u(\cdot, t)\|_{L^{2}}^{2}\left(-\varepsilon+\frac{\varepsilon}{2}\right) \\
& =-\varepsilon\|u(\cdot, t)\|_{L^{2}}^{2} .
\end{aligned}
$$

Therefore

$$
\begin{aligned}
\|u(\cdot, t)\|_{L^{2}} & \leq e^{-\varepsilon t}\|u(\cdot, 0)\|_{L^{2}} \\
& =e^{-\left(1-k_{2} \beta\right) t}\|u(\cdot, 0)\|_{L^{2}}
\end{aligned}
$$

which concludes the proof.

The next result generalizes Theorem 3.3 of [1].

Theorem 3.3. Suppose that (H1), (H3) hold and $k_{2} \beta<1$. Then there exists a global attractor $\mathcal{A}$ for the flow $T(t)$ generated by (2.6) in $L^{2}\left(S^{1}\right)$, which is contained in the ball of radius $\frac{2 \sqrt{2 \tau}\left(k_{2} \beta h+k_{3}\right)}{1-k_{2} \beta}$.

Proof If $u(w, t)$ is the solution of (2.6) with initial condition $u(w, 0)$ we have, by the variation of constants formula

$$
u(w, t)=e^{-t} u(w, 0)+\int_{0}^{t} e^{s-t} g(\beta(J * u(w, s)+h)) d s .
$$

Write

and

$$
T_{1}(t) u(w)=e^{-t} u(w, 0)
$$

$$
T_{2}(t) u(w)=\int_{0}^{t} e^{s-t} g(\beta(J * u(w, s)+h)) d s
$$

and suppose $u(\cdot, 0) \in C$, where $C$ is a bounded set in $L^{2}\left(S^{1}\right)$. Then

$$
\left\|T_{1}(t) u\right\|_{L^{2}} \longrightarrow 0 \text {, as } t \longrightarrow \infty \text {, uniformly in } u \text {. }
$$


Also, using (3.8), we have that $\|u(\cdot, t)\|_{L^{2}} \leq K$, for $t \geq 0$, where $K=$ $\max \left\{R, \frac{2 \sqrt{2 \tau}\left(k_{2} \beta h+k_{3}\right)}{1-k_{2} \beta}\right\}$. Therefore, for $t \geq 0$ we have

$$
\begin{aligned}
\frac{\partial T_{2}(t) u(w)}{\partial w} & =\int_{0}^{t} e^{s-t} \frac{\partial}{\partial w} g(\beta(J * u(w, s)+h)) d s \\
& =\beta \int_{0}^{t} e^{s-t} g^{\prime}(\beta(J * u(w, s)+h))\left(J^{\prime} * u\right)(w, s) d s .
\end{aligned}
$$

Thus

$$
\left|\frac{\partial T_{2}(t) u(w)}{\partial w}\right| \leq \beta \int_{0}^{t} e^{s-t}\left|g^{\prime}(\beta J * u(w, s)+\beta h) \|\left(J^{\prime} * u\right)(w, s)\right| d s .
$$

Using (H3) and (2.7), we obtain

$$
\begin{aligned}
\mid g^{\prime}(\beta J & * u(w, s)+\beta h) \|\left(J^{\prime} * u\right)(w, s) \mid \leq \\
& \leq\left[k_{4}|\beta J * u(w, s)+\beta h|+k_{5}\right]\left|J^{\prime} * u(w, s)\right| \\
& \leq\left[k_{4}|\beta J * u(w, s)|+k_{4} \beta h+k_{5}\right]\left|J^{\prime} * u(w, s)\right| \\
& \leq\left[k_{4} \beta \sqrt{2 \tau}\|J\|_{\infty}\|u(\cdot, s)\|_{L^{2}}+k_{4} \beta h+k_{5}\right] \sqrt{2 \tau}\left\|J^{\prime}\right\|_{\infty}\|u(\cdot, s)\|_{L^{2}} \\
& \leq k_{4} \beta 2 \tau\|J\|_{\infty}\left\|J^{\prime}\right\|_{\infty} K^{2}+\left(k_{4} \beta h+k_{5}\right) \sqrt{2 \tau}\left\|J^{\prime}\right\|_{\infty} K .
\end{aligned}
$$

Hence

$$
\begin{aligned}
& \left|\frac{\partial T_{2}(t) u(w)}{\partial w}\right| \leq \\
& \leq \beta \int_{0}^{t} e^{s-t}\left[k_{4} \beta 2 \tau\|J\|_{\infty}\left\|J^{\prime}\right\|_{\infty} K^{2}+\left(k_{4} \beta h+k_{5}\right) \sqrt{2 \tau}\left\|J^{\prime}\right\|_{\infty} K\right] d s \\
& =\left[k_{4} \beta^{2} 2 \tau\|J\|_{\infty}\left\|J^{\prime}\right\|_{\infty} K^{2}+\left(k_{4} \beta^{2} h+k_{5} \beta\right) \sqrt{2 \tau}\left\|J^{\prime}\right\|_{\infty} K\right] \int_{0}^{t} e^{s-t} d s \\
& \leq\left[k_{4} \beta^{2} 2 \tau\|J\|_{\infty}\left\|J^{\prime}\right\|_{\infty} K^{2}+\left(k_{4} \beta^{2} h+k_{5} \beta\right) \sqrt{2 \tau}\left\|J^{\prime}\right\|_{\infty} K\right] .
\end{aligned}
$$

It follows that, for $t>0$ and any $u \in C$, the value of $\left\|\frac{\partial T_{2}(t) u}{\partial w}\right\|_{L^{2}}$ is bounded by a constant (independent of $t$ and $u$ ). Thus, for all $u \in C$, we have that $T_{2}(t) u$ belongs to a ball of $W^{1,2}\left(S^{1}\right)$. From Sobolev's Imbedding Theorem, it follows that

$$
\bigcup_{t \geq 0} T_{2}(t) C
$$

is relatively compact. Therefore, the result follows from Theorem 3.1, the attractor $\mathcal{A}$ being the set $\omega$-limit of the ball $B\left(0, \frac{2 \sqrt{2 \tau}\left(k_{2} \beta h+k_{3}\right)}{1-k_{2} \beta}\right)$ in $L^{2}\left(S^{1}\right)$. 


\section{Comparison and boundedness results}

In this section we prove a comparison result that generalizes Theorem 2.7 of [8], where the case $g \equiv \tanh$ and $h=0$ was considered.

Definition 4.1. A function $v(w, t)$ is a subsolution of the Cauchy problem for (2.6) with initial condition $u(\cdot, 0)$ if $v(w, 0) \leq u(w, 0)$ for almost all $w \in S^{1}, v$ is continuously differentiable with respect to $t$ and satisfies

$$
\frac{\partial v(w, t)}{\partial t} \leq-v(w, t)+g(\beta(J * v(w, t)+h)),
$$

almost everywhere.

Analogously, the function $V(w, t)$ is a super solution if has the same regularity properties as above, satisfies (4.9) with reversed inequality and $V(w, 0) \geq u(w, 0)$ for almost all $w \in S^{1}$.

Theorem 4.2. (Comparison Theorem) Assume hypotheses (H1) and (H4) hold and let $v(w, t),[V(w, t)]$ be a sub solution [super solution] of the Cauchy problem of (2.6) with initial condition $u(\cdot, 0)$. Then

$$
v(w, t) \leq u(w, t) \leq V(w, t),
$$

almost everywhere.

\section{Proof}

Define the operator $G$ on $L^{\infty}\left(S^{1} \times[0, T]\right)$ by

$$
G(f)(w, t)=e^{-t} f(w, 0)+\int_{0}^{t} e^{-(t-s)} g(\beta(J * f(w, s)+h)) d s .
$$

Then $(G(f))(w, 0)=f(w, 0)$. Also, from (H4), it follows that $G$ is monotonic, that is, for any $f_{1}, f_{2} \in L^{\infty}\left(S^{1} \times[0, T]\right)$ with $f_{1} \geq f_{2}$ (a.e. in $\left.S^{1} \times[0, T]\right), G\left(f_{1}\right) \geq G\left(f_{2}\right)$ (a.e. in $\left.S^{1} \times[0, T]\right)$.

From (1.4), we obtain

$$
\begin{aligned}
& |G(f)(w, t)| \leq e^{-t}|f(w, 0)|+\int_{0}^{t} e^{-(t-s)}|g(\beta(J * f)(w, s)+\beta h)| d s \\
& \leq e^{-t}|f(w, 0)|+\int_{0}^{t} e^{-(t-s)}\left[k_{2}|\beta(J * f)(w, s)+\beta h|+k_{3}\right] d s \\
& \leq e^{-t}|f(w, 0)|+\int_{0}^{t} e^{-(t-s)} k_{2} \beta|(J * f)(w, s)| d s+ \\
& +\int_{0}^{t} e^{-(t-s)}\left(k_{2} \beta h+k_{3}\right) d s .
\end{aligned}
$$


Since $|(J * f)(w, s)| \leq\|f\|_{\infty}$ almost everywhere in $S^{1} \times[0, T]$, we obtain

$$
\begin{aligned}
& \|G(f)\|_{\infty} \leq \\
& \leq e^{-t}\|f\|_{\infty}+k_{2} \beta\|f\|_{\infty} \int_{0}^{t} e^{-(t-s)} d s+\left(k_{2} \beta h+k_{3}\right) \int_{0}^{t} e^{-(t-s)} d s \\
& \leq\|f\|_{\infty}+k_{2} \beta\|f\|_{\infty}+k_{2} \beta h+k_{3} .
\end{aligned}
$$

Therefore $G: L^{\infty}\left(S^{1} \times[0, T]\right) \rightarrow L^{\infty}\left(S^{1} \times[0, T]\right)$.

Furthermore, if $k_{1} \beta T<1, G$ is a contraction in any subset of functions of $L^{\infty}\left(S^{1} \times[0, T]\right)$ with the same values at $t=0$. In fact

$$
\begin{aligned}
& \left|G\left(f_{1}\right)(w, t)-G\left(f_{2}\right)(w, t)\right|= \\
& =\left|\int_{0}^{t} e^{-(t-s)}\left[g\left(\beta\left(J * f_{1}\right)(w, s)+\beta h\right)-g\left(\beta\left(J * f_{2}\right)(w, s)+\beta h\right)\right] d s\right| \\
& \leq \int_{0}^{t} e^{-(t-s)} k_{1} \beta\left|\left(J * f_{1}\right)(w, s)-\left(J * f_{2}\right)(w, s)\right| d s \\
& \leq \int_{0}^{t} e^{-(t-s)} k_{1} \beta\left(J *\left|f_{1}-f_{2}\right|(w, s)\right) d s \\
& \leq \int_{0}^{t} e^{-(t-s)} k_{1} \beta J *\left\|f_{1}-f_{2}\right\|_{\infty} d s \\
& =k_{1} \beta T\left\|f_{1}-f_{2}\right\|_{\infty} \int_{0}^{t} e^{-(t-s)} d s \\
& \leq k_{1} \beta T\left\|f_{1}-f_{2}\right\|_{\infty},
\end{aligned}
$$

almost everywhere in $S^{1} \times[0, T]$. Hence

$\left\|G\left(f_{1}\right)-G\left(f_{2}\right)\right\|_{\infty} \leq k_{1} \beta T\left\|f_{1}-f_{2}\right\|_{\infty}$. Therefore, if $k_{1} \beta T<1, G$ is a contraction. Thus, if $u(w, t)$ is a solution of $(2.6)$ with $u^{0}=u(w, 0)$, we have

$$
u=\lim _{n \longrightarrow \infty} G^{n}\left(u^{0}\right)
$$

on $L^{\infty}\left(S^{1} \times[0, T]\right)$. The same holds for a solution $\widetilde{u}$ with $\widetilde{u}^{0}=\widetilde{u}(w, 0)$. If $\widetilde{u}^{0} \leq u^{0}$ a.e., with $g$ monotonic, it follows that

$$
G^{n}\left(\widetilde{u}^{0}\right) \leq G^{n}\left(u^{0}\right) \text {, a.e. }
$$

Now, if $v$ is a sub solution of (2.6) we have

$$
\frac{d}{d t} v(w, t)+v(w, t) \leq g(\beta(J * v(w, t)+h)), \text { a.e. }
$$

Multiplying both sides of the inequality above by $e^{t}$, we have

$$
\frac{d}{d t}\left(e^{t} v(w, t)\right) \leq e^{t} g(\beta(J * v(w, t)+h)) \text {, a.e. }
$$


Integrating from 0 to $t$, we obtain

$$
v(w, t) \leq e^{-t} v(w, 0)+\int_{0}^{t} e^{-(t-s)} g(\beta(J * v(w, s)+h)) d s,
$$

almost everywhere. Therefore $v(w, t) \leq G(v)(w, t)$, a.e., and since $g$ monotonic, it follows that $v(w, t) \leq G^{n}(v)(w, t)$ almost everywhere. Thus, $v(w, t) \leq z(w, t)$, a.e., where

$$
z=\lim _{n \rightarrow \infty} G^{n+1}(v) .
$$

Now, from the continuity of $G$, it follows that

$$
G(z)=G\left(\lim _{n \longrightarrow \infty} G^{n}(v)\right)=\lim _{n \longrightarrow \infty} G^{n+1}(v)=z .
$$

Therefore $z$ is a fixed point of $G$, that is, $z$ is a solution of $(2.6)$ in $S^{1} \times[0, T]$ with initial condition $z(\cdot, 0)=v(\cdot, 0)$. Thus, if $z(\cdot, 0) \leq u(\cdot, 0)$, a.e., then

$$
v \leq z \leq u, \text { a.e. in } S^{1} \times[0, T],
$$

where $u$ is the solution of $(2.6)$ with initial condition $u(\cdot, 0)$. If $V(w, t)$ is a super solution we obtain, by the same arguments

$$
u \leq \widetilde{z} \leq V \text {, a.e. in } S^{1} \times[0, T] .
$$

Therefore

$$
v(w, t) \leq u(w, t) \leq V(w, t),
$$

almost everywhere in $S^{1} \times[0, T]$.

Since the estimates above do not depend on the initial condition, we may extend the result to $[T, 2 T]$ and, by iteration, we can complete the proof of the theorem.

Remark 4.3. If we add the hypothesis (H5), with $a<\infty$, the comparison result holds in the ball $\mathbb{M}=\left\{L^{\infty}\left(S^{1} \times[0, T]\right),\|\cdot\|_{\infty} \leq a\right\}$.

In fact, it is enough to prove that $\left.G\right|_{\mathbb{M}}: \mathbb{M} \rightarrow \mathbb{M}$. But, from (H4), it follows that

$$
\left|\left(\left.G\right|_{\mathbb{M} f} f\right)(w, t)\right| \leq e^{-t}|f(w, 0)|+a \int_{0}^{t} e^{-(t-s)} d s .
$$

Hence

$$
\begin{aligned}
\left\|\left(\left.G\right|_{\mathbb{M}} f\right)\right\|_{\infty} & \leq e^{-t}\|f\|_{\infty}+a \int_{0}^{t} e^{-(t-s)} d s \\
& \leq a e^{-t}+a \int_{0}^{t} e^{-(t-s)} d s \\
& =a .
\end{aligned}
$$


Therefore, $\left.G\right|_{\mathbb{M}}(f) \in \mathbb{M}$.

Theorem 4.4. Assume the hypotheses (H1) and (H5) with $a<\infty$. Then the attractor $\mathcal{A}$ belongs to the ball $\|\cdot\|_{\infty} \leq$ a in $L^{\infty}\left(S^{1}\right)$.

Proof Since the hypothesis (H5) is a particular case of (1.4) with $k_{2}=0$ and $k_{3}=a$, it follows from Theorem 3.3 that the attractor is contained in the ball $B[0,2 a \sqrt{2 \tau}]$ in $L^{2}\left(S^{1}\right)$.

Let $u(w, t)$ be a solution of $(2.6)$ in $\mathcal{A}$. Then, by the variation of constants formula

$$
u(w, t)=e^{-\left(t-t_{0}\right)} u\left(w, t_{0}\right)+\int_{t_{0}}^{t} e^{-(t-s)} g(\beta(J * u)(w, s)+\beta h) d s .
$$

Since $\|u\|_{L^{2}} \leq 2 a \sqrt{2 \tau}$ for all $u \in \mathcal{A}$, we obtain for all $(w, t) \in S^{1} \times \mathbb{R}^{+}$ letting $t_{0} \rightarrow-\infty$

$$
u(w, t)=\int_{-\infty}^{t} e^{-(t-s)} g(\beta(J * u)(w, s)+\beta h) d s
$$

where the equality above is in the sense of $L^{2}\left(S^{1}\right)$. Thus, using (H5) again, we have

$$
\begin{aligned}
|u(w, t)| & \leq \int_{-\infty}^{t} e^{-(t-s)}|g(\beta(J * u)(w, s)+\beta h)| d s \\
& \leq \int_{-\infty}^{t} a e^{-(t-s)} d s \\
& \leq a
\end{aligned}
$$

as claimed.

\section{Existence of a Lyapunov functional}

In this section we exhibit a continuous "Lyapunov's functional" for the flow of (2.6), restricted to the ball of radius $a$ in $L^{\infty}\left(S^{1}\right)$, concluding that this flow is gradient, in the sense of [5].

We claim that $\left\{L^{2}\left(S^{1}\right),\|\cdot\|_{\infty} \leq a\right\}$ is an invariant set for the flow generated by (2.6) . In fact, if $a=\infty$, there is nothing to prove. Otherwise, let

$$
u(w, t)=e^{-t} u(w, 0)+\int_{0}^{t} e^{-(t-s)} g(\beta J * u(w, s)+\beta h) d s
$$


be the solution of (2.6) with initial condition $u(w, 0) \in\left\{L^{2}\left(S^{1}\right),\|\cdot\|_{\infty} \leq a\right\}$. Then

$$
\begin{aligned}
|u(w, t)| & \leq e^{-t}|u(w, 0)|+\int_{0}^{t} e^{-(t-s)}|g(\beta J * u(w, s)+\beta h)| d s \\
& \leq e^{-t}|u(w, 0)|+a \int_{0}^{t} e^{-(t-s)} d s .
\end{aligned}
$$

Hence

$$
\begin{aligned}
\|u(\cdot, t)\|_{\infty} & \leq e^{-t}\|u(\cdot, 0)\|_{\infty}+a \int_{0}^{t} e^{-(t-s)} d s \\
& \leq e^{-t} a+a \int_{0}^{t} e^{-(t-s)} d s \\
& =a .
\end{aligned}
$$

Define the functional $\mathbb{F}:\left(L^{2}\left(S^{1}\right),\|u\|_{\infty} \leq a\right) \rightarrow \mathbb{R}$ by

$\mathbb{F}(u)=\int_{S^{1}}[f(u(w))-f(\bar{m})] d w+\frac{1}{4} \int_{S^{1}} \int_{S^{1}} J\left(w \cdot z^{-1}\right)[u(w)-u(z)]^{2} d w d z$,

where $f$ is given in the hypothesis ( $\mathrm{H} 6)$.

Note that, if $a<\infty$, the functional in (5.10) is defined in the whole space $\left\{L^{2}\left(S^{1}\right),\|u\|_{\infty} \leq a\right\}$. This is not true for the similar functional

$$
\widetilde{\mathbb{F}}(u)=\int_{\mathbb{R}}\left[f(u(w))-f\left(m_{\beta}^{+}\right)\right] d w+\frac{1}{4} \int_{\mathbb{R}} \int_{\mathbb{R}} J(w-z)[u(w)-u(z)]^{2} d w d z,
$$

considered in [7], [8] and [11] with $g \equiv$ tanh.

It is proved in [8], in the case of unbounded domain, $g \equiv \tanh$ and $h=0$, that the functional $\widetilde{\mathbb{F}}$ is lower semicontinuous in the weak $L_{l o c}^{2}$ topology. In our case, however, a stronger continuity property can be proved.

Theorem 5.1. Assume (H6) holds with $a<\infty$. Then the functional given in (5.10) is continuous in the topology of $L^{2}\left(S^{1}\right)$.

Proof Note that, if $\|u\|_{\infty} \leq a$, there exists a positive constant $K$ such that

$$
|f(u(w))-f(\bar{m})| \leq|f(u(w))|+|f(\bar{m})| \leq K, \text { for almost every } w \in S^{1} .
$$

for any $u \in L^{2}\left(S^{1}\right)$, with $\|u\|_{\infty} \leq a$, let $u_{n}$ a sequence converging to $u$ in the norm of $L^{2}\left(S^{1}\right)$. We can extract a subsequence $u_{n_{k}}$, such that, $u_{n_{k}}(w) \longrightarrow u(w)$ a.e. in $S^{1}$. Since from (H6), it follows that $f$ is continuous, $f\left(u_{n_{k}}(w)\right) \longrightarrow f(u(w))$ a.e. Thus

$$
\lim _{k \longrightarrow \infty}\left[f\left(u_{n_{k}}(w)\right)-f(\bar{m})\right]=[f(u(w))-f(\bar{m})] \text {, a.e. }
$$


and

$$
\lim _{k \longrightarrow \infty}\left[u_{n_{k}}(w)-u_{n_{k}}(z)\right]^{2}=[u(w)-u(z)]^{2}, \text { a.e. }
$$

Now, we write

$$
\mathbb{F}(u)=\mathbb{F}_{1}(u)+\mathbb{F}_{2}(u),
$$

where

$$
\mathbb{F}_{1}=\int_{S^{1}}[f(u(w))-f(\bar{m})] d w
$$

and

Since

$$
\mathbb{F}_{2}(u)=\frac{1}{4} \int_{S^{1}} \int_{S^{1}} J\left(w \cdot z^{-1}\right)[u(w)-u(z)]^{2} d w d z .
$$

$$
\left|f\left(u_{n_{k}}(w)\right)-f(\bar{m})\right| \leq K,
$$

we can apply Lebesgue's Dominated Convergence Theorem to obtain

$$
\lim _{k \longrightarrow \infty} \mathbb{F}_{1}\left(u_{n_{k}}\right)=\mathbb{F}_{1}(u) \text {. }
$$

Similarly, as

$$
\left|u_{n_{k}}(w)-u_{n_{k}}(z)\right|^{2} \leq 4 a^{2} \in L^{1}\left(S^{1}\right),
$$

we have

$$
\lim _{k \rightarrow \infty} \mathbb{F}_{2}\left(u_{n_{k}}\right)=\mathbb{F}_{2}(u) \text {. }
$$

Therefore

$$
\lim _{k \longrightarrow \infty} \mathbb{F}\left(u_{n_{k}}\right)=\mathbb{F}(u)
$$

Thus $\mathbb{F}\left(u_{n}\right)$ is a sequence such that every subsequence has a subsequence that converges to $\mathbb{F}(u)$, and we obtain

$$
\lim _{n \rightarrow \infty} \mathbb{F}\left(u_{n}\right)=\mathbb{F}(u) .
$$

Theorem 5.2. Suppose that the hypotheses (H1), (H4) and (H5)-(H6), with $a<\infty$, hold. Let $u(\cdot, t)$ be a solution of (2.6) with $u(\cdot, t) \leq a$. Then $\mathbb{F}(u(\cdot, t))$ is differentiable with respect to $t$ for $t>0$ and

$$
\frac{d}{d t} \mathbb{F}(u(\cdot, t))=-I(u(\cdot, t)) \leq 0,
$$

where, for any $u \in L^{2}\left(S^{1}\right)$ with $\|u\|_{\infty} \leq a$,

$I(u(\cdot))=\int_{S^{1}}\left[(J * u)(w)+h-\beta^{-1} g^{-1}(u(w))\right][g(\beta(J * u)(w)+\beta h)-u(w)] d w$.

Furthermore, the integrand in $I(u(\cdot))$ is a non negative function and, $u$ is a critical point of $\mathbb{F}$ if only if $u$ is an equilibrium of (2.6). 
Proof From (H1) and (H5), it follows that $\mathbb{F}(u(\cdot, t))$ is well defined for all $t \geq 0$. We assume first that, given $t>0$, there exists $\varepsilon>0$ such that $\|u(\cdot, s)\|_{\infty} \leq a-\varepsilon$, for $s \in \Delta$ where $\Delta$ is a closed finite interval containing $t$. For $s \in \Delta$ we write

$$
\mathbb{F}(u(\cdot, s))=\int_{S^{1}} \phi(w, s) d w, \text { and } I(u(\cdot, s))=\int_{S^{1}} \iota(w, s) d w .
$$

As

$$
\begin{aligned}
& \frac{\partial \phi}{\partial s}(w, s)= \\
& {\left[-u(w, s)-h+\beta^{-1} g^{-1}(u(w, s))\right][-u(w, s)+g(\beta((J * u)(w, s)+h))]} \\
& +\frac{1}{2} \int_{S^{1}} J\left(w \cdot z^{-1}\right)[u(w, s)-u(z, s)]\left[\frac{\partial u(w, s)}{\partial s}-\frac{\partial u(z, s)}{\partial s}\right] d z,
\end{aligned}
$$

$\frac{\partial \phi(w, s)}{\partial s}$ is almost everywhere continuous and bounded in $w$ for $s \in \Delta$, that is,

$$
\sup _{s \in \Delta}\left\|\frac{\partial \phi(\cdot, s)}{\partial s}\right\|_{L^{1}}<\infty .
$$

Therefore, we can derive under the integration sign obtaining

$$
\begin{aligned}
\frac{d}{d s} \mathbb{F}(u(\cdot, s)) & =\int_{S^{1}}\left[-u(w, s)-h+\beta^{-1} g^{-1}(u(w, s))\right] \frac{\partial u(w, s)}{\partial s} d w \\
& +\frac{1}{2} \int_{S^{1}} \int_{S^{1}} J\left(w \cdot z^{-1}\right)[u(w, s)-u(z, s)] \\
& *\left[\frac{\partial u(w, s)}{\partial s}-\frac{\partial u(z, s)}{\partial s}\right] d w d z
\end{aligned}
$$


Since

$$
\begin{array}{rl}
\int_{S^{1}} \int_{S^{1}} & J\left(w \cdot z^{-1}\right)[u(w, s)-u(z, s)]\left[\frac{\partial u(w, s)}{\partial s}-\frac{\partial u(z, s)}{\partial s}\right] d w d z= \\
= & \int_{S^{1}} \int_{S^{1}} J\left(w \cdot z^{-1}\right) u(w, s) \frac{\partial u(w, s)}{\partial s} d w d z \\
& -\int_{S^{1}} \int_{S^{1}} J\left(w \cdot z^{-1}\right) u(w, s) \frac{\partial u(z, s)}{\partial s} d w d z \\
& -\int_{S^{1}} \int_{S^{1}} J\left(w \cdot z^{-1}\right) u(z, s) \frac{\partial u(w, s)}{\partial s} d w d z \\
& +\int_{S^{1}} \int_{S^{1}} J\left(w \cdot z^{-1}\right) u(z, s) \frac{\partial u(z, s)}{\partial s} d w d z \\
= & 2 \int_{S^{1}} \int_{S^{1}} J\left(w \cdot z^{-1}\right) u(w, s) \frac{\partial u(w, s)}{\partial s} d w d z \\
& -2 \int_{S^{1}} \int_{S^{1}} J\left(w \cdot z^{-1}\right) u(z, s) \frac{\partial u(w, s)}{\partial s} d w d z .
\end{array}
$$

and

it follows that

$$
\begin{aligned}
\frac{d}{d s} \mathbb{F}(u(\cdot, s)) & =\int_{S^{1}}\left[-u(w, s)-h+\beta^{-1} g^{-1}(u(w, s))\right] \frac{\partial u(w, s)}{\partial s} d w \\
& +\int_{S^{1}}\left(\int_{S^{1}} J\left(w \cdot z^{-1}\right) d z\right) u(w, s) \frac{\partial u(w, s)}{\partial s} d w \\
& -\int_{S^{1}}\left(\int_{S^{1}} J\left(w \cdot z^{-1}\right) u(z, s) d z\right) \frac{\partial u(w, s)}{\partial s} d w \\
& =\int_{S^{1}}\left[-u(w, s)-h+\beta^{-1} g^{-1}(u(w, s))\right] \frac{\partial u(w, s)}{\partial s} d w \\
& +\int_{S^{1}}[u(w, s)-(J * u)(w, s)] \frac{\partial u(w, s)}{\partial s} d w \\
& =\int_{S^{1}}\left[-(J * u)(w, s)-h+\beta^{-1} g^{-1}(u(w, s))\right] \\
& *[-u(w, s)+g(\beta(J * u(w, s)+h))] d w \\
& =-I(u(\cdot, s)) .
\end{aligned}
$$


This proves the first part of theorem with the additional hypothesis that $\|u(\cdot, s)\|_{\infty} \leq a-\varepsilon$, for $s \in \Delta$ and some $\varepsilon>0$, where $\Delta$ is a closed finite interval containing $t$. We claim that this hypothesis actually holds for all $t>0$.

Let $\lambda(w, t)$ be the solution of (2.6) such that $\lambda(w, 0)=a$ for any $w \in S^{1}$. Then $\lambda(w, t)=\lambda(t)$ where

$$
\frac{d \lambda}{d t}=-\lambda(t)+g(\beta(\lambda(t)+h)) .
$$

Since by hypothesis (H5), $|g(x)|<a, \forall x \in \mathbb{R}$, it follows easily that $\lambda(t)<a$ for any $t>0$. Since $u(w, 0) \leq a$, we obtain by the Comparison Theorem

$$
u(w, t) \leq \lambda(t)<a,
$$

for almost every $w \in S^{1}$ and $t>0$. Repeating the same argument, starting from inequality $u(w, 0) \geq-a$, for almost every $w \in S^{1}$, we obtain $u(w, t) \geq-\lambda(t)>-a$, and thus

$$
\|u(\cdot, t)\|_{\infty}<\lambda(t)<a, \quad \text { for all } t>0
$$

and the claim follows by continuity.

To conclude the proof, it is enough to show that $u$ is a critical point of $\mathbb{F}$ if only if $u$ is an equilibrium of (2.6). Let $u(w)$ be a critical point of the functional $\mathbb{F}$, then $I(u(\cdot))=0$. Since the integrand is non negative almost everywhere, it follows that

$$
\left[(J * u(w))+h-\beta^{-1} g^{-1}(u(w))\right][g(\beta(J * u(w)+h))-u(w)]=0
$$

almost everywhere. But the annihilation of any of these factors implies

$$
g(\beta(J * u(w)+h))=u(w) .
$$

Reciprocally, if $u$ is a equilibrium of (2.6), it is easy to see that $I(u(\cdot))=0$.

As a immediate consequence of the existence of the functional $\mathbb{F}$ we obtain the following result.

Corollary 5.3. There are no non trivial recurrent points under the flow of (2.6).

Remark 5.4. The integrand in the functional $\mathbb{F}$ above is always non negative since $J$ is positive and $\bar{m}$ is a global minim of $f$. Thus $\mathbb{F}$ is lower bounded.

We recall that a $C^{r}$-semigroup, $T(t)$, is gradient if each bounded positive orbit is precompact and there exists a Lyapunov Functional for $T(t)$, (see [5]).

Proposition 5.5. Assume the hypotheses (H1), (H3), (H4) and (H5), (H6) with $a<\infty$. Then the flow generated by equation (2.6) is gradient. 
Proof The precompacity of the orbits follows from the existence of the global attractor. From Theorems 5.1 and 5.2, and Remark 5.4, we have existence of a continuous Lyapunov functional.

As a consequence of the existence of the Lyapunov functional, we have the following characterization of the attractor (see [5] - Theorem 3.8.5).

Theorem 5.6. Assume the same assumptions of Proposition 5.5. Then the attractor $\mathcal{A}$ is the unstable set of the equilibrium point set of $T(t)$, that is,

$$
\mathcal{A}=W^{u}(E) .
$$

\section{References}

[1] Barros, S.R.M., Pereira, A.L., Possani, C., Simonis, A., Spatial Periodic Equilibria for a Non local Evolution Equation. Discrete and Continuous Dynamical Systems 9 N. 4, (2003), 937-948.

[2] Brezis, H., Analisis funcional, teoria y aplicaciones. Alianza, Madrid, 1984.

[3] Daleckii, J.L., Krein, M.G., Stability of Solutions of Differential Equations in Banach Spaces. American Mathematical Society, Providence, Rhode Island, 1974.

[4] Folland, G., Introduction to partial differential equations. Princeton Univ. Press, 1976.

[5] Hale, J.K., Asymptotic behavior of dissipative Systems. American Surveys and Monographs, N. 25, 1988.

[6] Henry, D., Geometric Theory of Semilinear Parabolic Equations. Lecture Notes in Mathematics N. 840, Springer-Verlag, 1981.

[7] Masi, A., Gobron, T., Presutti, E., Traveling fronts in non local evolution equations. Arch. Rational Mech. Anal. 132 (1995), 143-205.

[8] Masi, A., Orland, E., Presutti, E., Triolo, L., Uniqueness and global stability of the instanton in non local evolution equations. Rendiconti di Matematica, Serie VII, Vol. 14, (1994), 693-723.

[9] Masi, E., Oliveri, E., Presutti, E., Critical droplet for a non local mean field equation. Markov Processes Relat. Fields 6 (2000), 439-471.

[10] Masi, A., Orland, E., Presutti, E., Triolo, L., Stability of the interface in a model of phase separation. Proc. Royal Society of Edinburgh 124A, (1994), 1013-1022.

[11] Pereira, A.L., Global attractor and nonhomogeneous equilibria for a non local evolution equation in an unbounded domain. J. Diff. Equations 226 (2006) 352372.

[12] Rall, L.B., Nonlinear Functional Analysis and Applications. Academic Press, New York-London, 1971.

[13] Teman, R., Infinite Dimensional Dynamical Systems in Mechanics and Physics. Springer, 1988. 